\title{
Facile fabrication of functional cellulose paper with high-capacity immobilization of Ag nanoparticles for catalytic applications for tannery wastewater
}

\author{
Ruiquan $\mathrm{Yu}^{1 \dagger}$, Tianxiang Lan ${ }^{1 \dagger}$, Jing Jiang ${ }^{2}$, Hao Peng ${ }^{1}$, Ruifeng Liang ${ }^{3}$ and Gongyan Liu ${ }^{1,2^{*}}$
}

\begin{abstract}
It has been a research goal to develop macroscopic materials with an optimized surface structure to affix silver nanoparticles which could contaminate water and maximize their practical functions. Cellulose paper is a versatile biomass material valued for its abundance, low cost, biocompatibility, and natural composition. Until now, its potential application in water purification has not been adequately explored. In this study, gallic acid-modified silver nanoparticles (GA@AgNPs) were loaded onto commercial cellulose filter paper using a simple lipoic acid modification process (GA@AgNPs-LA-CP). Scanning electron microscopy (SEM), X-ray photoelectron spectroscopy (XPS), and X-ray diffraction (XRD) were used to characterize the GA@AgNPs-LA-CP. The catalytic activity of the GA@AgNPs-LA-CP was evaluated by the reduction reaction of methylene blue (MB), Rhodamine B (RhB), and 4nitrophenol (4-NP) with sodium borohydride $\left(\mathrm{NaBH}_{4}\right)$. The GA@AgNPs-LA-CP exhibited excellent catalytic activity toward MB, RhB, and 4-NP, taking advantage of its high specific surface area generated by the cellulose fiber network structure. Interestingly, due to the electrostatic interactions between the cationic dyes and the GA@AgNPs, the as-prepared catalytic composite material serves as a better catalyst for MB and RhB, suggesting dual applications of the composite materials for organic wastewater treatment and the removal of harmful dyes. This implies that the immobilization of AgNPs on cellulose papers is an effective method and can be applied to efficient wastewater treatment applications.
\end{abstract}

Keywords: Ag nanoparticles, Catalyst, Tannery wastewater, Cellulose paper

\section{Introduction}

Pollution in large bodies of water has become a serious environmental problem [1, 2]. In many organic pollutants, there are significant health issues due to the extensive application of organic dyes in leather [3], paper [4], textiles [5], printing [6], and the paint [7] and plastics

\footnotetext{
* Correspondence: lgy3506@scu.edu.cn

${ }^{+}$Ruiquan Yu and Tianxiang Lan contributed equally to this work.

${ }^{1}$ National Engineering Laboratory for Clean Technology of Leather

Manufacture, College of Biomass Science and Engineering, Sichuan

University, Chengdu 610065, China

${ }^{2}$ The Key Laboratory of Leather Chemistry and Engineering of Ministry of Education, College of Biomass Science and Engineering, Sichuan University,

Chengdu 610065, China

Full list of author information is available at the end of the article
}

industries [8], it is particularly important to solve the pollution problem of organic dyes [9]. Uncontrolled releases of wastewater containing organic dyes prevents sunlight from penetrating into the water, thus reducing the photosynthetic response of plants [10]. In general, water can change colors at dye concentrations as low as $1 \mathrm{ppm}[11,12]$. So, removal of organic dyes in wastewater treatment is critical. Specifically, 4-nitrophenol (4$\mathrm{NP}$ ) is a kind of nitroaromatic compound that is difficult to degrade. It is listed by the U.S. Environmental Protection Agency (EPA) as one of the most important 114 organic pollutants [13, 14]. Meanwhile, these aromatic contaminants pose immediate and potential toxicity to 
aquatic plants and animals and microorganisms $[15,16]$. Furthermore, some aromatic pollutants can also cause carcinogenic and teratogenic effects [17]. Hence, various organic pollutant removal technologies has been developed, including adsorption [18, 19], membrane filtration [20], photocatalytic degradation [21, 22], chemical oxidation [23], flocculation [24] and electrooxidation [25]. Catalytic technology has been widely studied because of its high efficiency, simple operation, low cost, and wide range of applications $[26,27]$. Among these methods, the reduction of 4-NP to 4aminophenol (4-AP) by sodium borohydride is the most effective and environmentally friendly method [28]. 4-AP is an important intermediate for the synthesis of corrosion inhibitors and developers and analgesic and antipyretic drugs [29]. However, this relatively simple and clean reaction progresses slowly without a suitable catalyst. In addition, silver nanoparticles (AgNPs) are effective catalysts for the conversion of 4$\mathrm{NP}$ to 4-AP [30, 31]. Before 4-NP is discharged into the environment, it is necessary to develop a new catalyst with good dispersion and stable catalytic performance.

With the rapid development of modern nanotechnology [32, 33], silver [34], gold [35], and other nanoscale precious metal particles [36] have been widely used as nanocatalysts, which have aroused great interest. AgNPs have high catalytic activity and are widely used in the reduction and degradation of organic compounds [37, 38]. However, virgin AgNPs tend to aggregate in the catalytic process, thus inhibiting its catalytic performance and practical application [39]. To overcome this shortcoming, the immobilization of silver nanoparticles on a support material has been considered as an effective method to improve the stability of AgNPs, which is conducive to maintaining the catalytic ability of AgNPs [40, 41].

In recent years, biomass materials have been used as Ag nanoparticle carriers due to their low cost and easy availability, compared to other matrix carriers such as graphene and carbon nanotubes [42, 43]. In addition, biomass materials also have many advantageous properties, such as excellent thermal stability and chemical durability, high specific surface area, and rich pores and functional groups [44]. These important characteristics enable excellent nanocatalytic support material. Liang and his group [45] reported that AgNPs on a naturally fixed eggshell membrane (ESM) had good catalytic activity through proanthocyanidins (Pro) and AgNPs@proesm composites. Due to the interwoven fiber structure, the reduction of 4-NP is highly reusable. Although ESM has shown great potential as an immobilized biomass material of nanocatalysts, biomass materials with interwoven fibrous three-dimensional structures are rare in nature, difficult to collect, and challenging to process. These defects will restrict its large-scale application. Zeng and his group [15] reported facile fabrication of silver nanoparticles deposited with a poly-dopamine- coated magnetic biochar (MC-PDA-Ag) catalyst. The catalytic reduction experiments confirmed that MCPDA-Ag catalysts had good catalytic properties on model dyes (MB, RhB, and MO). However, it is not easy to recover these nanocomposites from solution due to the material instability.

In this work, a convenient lipoic acid modification method was developed to immobilize AgNPs on cellulose paper to catalyze harmful 4-NP into a more environmentally friendly 4-AP. In previous work [42], a negatively charged gallic acidmodified silver nanoparticles (GA@AgNPs) was synthesized with a large number of carboxyl groups on the surface and an average particle size of $8 \mathrm{~nm}$ and was used as a nanocatalyst. Lipoic acid modification on the surface of cellulose fibers could drive uniform immobilization of GA@AgNPs. In addition, two dyes, methylene blue (MB) and Rhodamine B $(\mathrm{RhB})$, were also tested in this study. The catalytic experiment showed that GA@AgNPs-LA-CP had good catalytic performance, which verified the immobilization of GA@AgNPs on LA-CP. The catalytic properties of GA@AgNPs-LA-CP are primarily determined by the silver nanoparticles on its surface. Based on the electrostatic interaction between cationic dyes $(\mathrm{MB}, \mathrm{RhB})$ and negatively charged GA@AgNPs-LA-CP, the GA@AgNPs-LA-CP showed excellent catalytic effects on cationic dyes. More importantly, the GA@AgNPs-LA-CP prepared in this study show great potential in large-scale practical applications for the removal of organic dyes and 4-NP from leather organic wastewater.

\section{Experimental section}

\subsection{Materials}

Lipoic acid (99\%), N, N'-dicyclohexylcarbodiimide (DCC, 99\%), 4-(dimethylamino) pyridine (DMAP, 99\%) and dimethylsulfoxide (DMSO, 99\%) was purchased from Aladdin Technology Co. Ltd. (Shanghai, China). Sodium borohydride $\left(\mathrm{NaBH}_{4},>96 \%\right)$, $p$-nitrophenol (4$\mathrm{NP})$, methylene blue (MB) and Rhodamine $\mathrm{B}(\mathrm{RhB})$ are purchased from Jinshan Chemical Reagents Corporation (Chengdu, China). Cellulose filter papers were purchased from Whatman (Shanghai, China). All other chemicals were analytical reagents.

\subsection{Preparation of lipoic acid-modified cellulose paper (LA-CP)}

A cellulose filter paper $(0.5 \mathrm{~g})$ was immersed in an aqueous solution of $10 \mathrm{wt} \% \mathrm{NaOH}(10 \mathrm{~mL})$ for $18 \mathrm{~h}$. The sample was then repeatedly washed with absolute ethanol until a solution was obtained around neutral $\mathrm{pH}$. The paper was used for further reactions after the sogginess of the paper was removed by air. A cellulose filter paper $(0.5 \mathrm{~g}, 9.1 \mathrm{mmol}$ of active $-\mathrm{OH}$ groups), 4(dimethylamino) pyridine $(0.15 \mathrm{~g}, 1.23 \mathrm{mmol}), \mathrm{N}, \mathrm{N}$ '-dicyclohexylcarbodiimide $(0.15 \mathrm{~g}, \quad 0.73 \mathrm{mmol})$ and lipoic acid $(0.24 \mathrm{~g}, 1.16 \mathrm{mmol})$ were added in DMSO (20 
$\mathrm{mL})$, respectively. The reaction beaker was shaken in a rotary shaker $(100 \mathrm{rpm})$ for $48 \mathrm{~h}$ under nitrogen at $30^{\circ} \mathrm{C}$. The product was isolated by precipitation in cold ethanol, washed several times with ethanol, and dried in vacuo. Lipoic acid modification of the cellulose paper was carried out by coupling the $-\mathrm{COOH}$ group of lipoic acid with the $-\mathrm{OH}$ group of the cellulose paper in the presence of DCC and DMAP.

\subsection{Preparation of GA@AgNPs immobilized cellulose} paper by lipoic acid modification (GA@AgNPs-LA-CP)

Gallic acid-modified silver nanoparticles (GA@AgNPs) with concentration of $100 \mathrm{mg} / \mathrm{L}$ were prepared according to our previous work [42]. Briefly, aqueous $\mathrm{AgNO}_{3}$ solution $(17 \mathrm{mM})$ was mixed with an equal volume of GA solution $(18 \mathrm{mM})$. Then, the above mixture was added into $\mathrm{NaBH}_{4}$ solution $(37 \mathrm{mM})$ and reacted for $2 \mathrm{~h}$ to obtain GA@AgNPs with average diameter of $8 \mathrm{~nm}$ and concentration of $400 \mathrm{ppm}$. The LA-CP samples were then immersed in the GA@AgNPs solution, and the reaction beaker was shaken in a rotary shaker $(100 \mathrm{rpm})$ for $30 \mathrm{~min}$ at room temperature. The brown paper (GA@AgNPsLA-CP) was washed with deionized water until the washing liquid appeared clear. The brown papers decorated with AgNPs were then dried in air and stored in a desiccator.

\subsection{Characterizations}

The surface morphologies of the synthesized GA@AgNPs-LA-CP samples were accomplished using field emission scanning electron microscopy (FESEM, JEOL, Japan) operated at an accelerating voltage of $15 \mathrm{kV}$. The elemental compositions of the synthesized paper samples were determined by an energy dispersed X-ray spectrometer (EDS) coupled with the FESEM. The chemical states of the element on the paper surface were investigated by X-ray photoelectron spectroscopy (XPS, XSAM800, Kratos Ltd., U.K.). Wide-angle X-ray diffraction (XRD) patterns were recorded at ambient temperature on a Philips X' Pert pro MPD diffractometer with $\mathrm{Cu}-\mathrm{K} \alpha$ radiation in the $2 \theta$ range from 30 to $80^{\circ}$. The surface zeta-potential value of samples was detected using the streaming potential method via an electrokinetic analyzer (Mütek SZP-10, BTG, Germany) with $1 \mathrm{mmol} / \mathrm{L} \mathrm{KCl}$ solution electrolyte solution. $0.05 \mathrm{M} \mathrm{NaOH}$ and $0.05 \mathrm{M} \mathrm{HCl}$ solutions were used to adjust the $\mathrm{pH}$ during the measurement. Ultraviolet-visible diffuse reflectance spectra (UVvis DRS) were recorded by means of UV-vis-NIR spectrophotometer (UV-3600, Shimadzu, Japan) equipped with an integrating sphere and using $\mathrm{BaSO}_{4}$ as reference.

\subsection{Measurement of Ag content on GA@AgNPs-LA-CP}

To quantify the amount of silver in the GA@AgNPs-LA-CP, we performed an acid digestion of the paper and analyzed the amount of dissolved silver with an inductively coupled plasma atomic emission spectrometer (ICP-AES, model JY38S, France). Briefly, approximately $100 \mathrm{mg}$ of dried

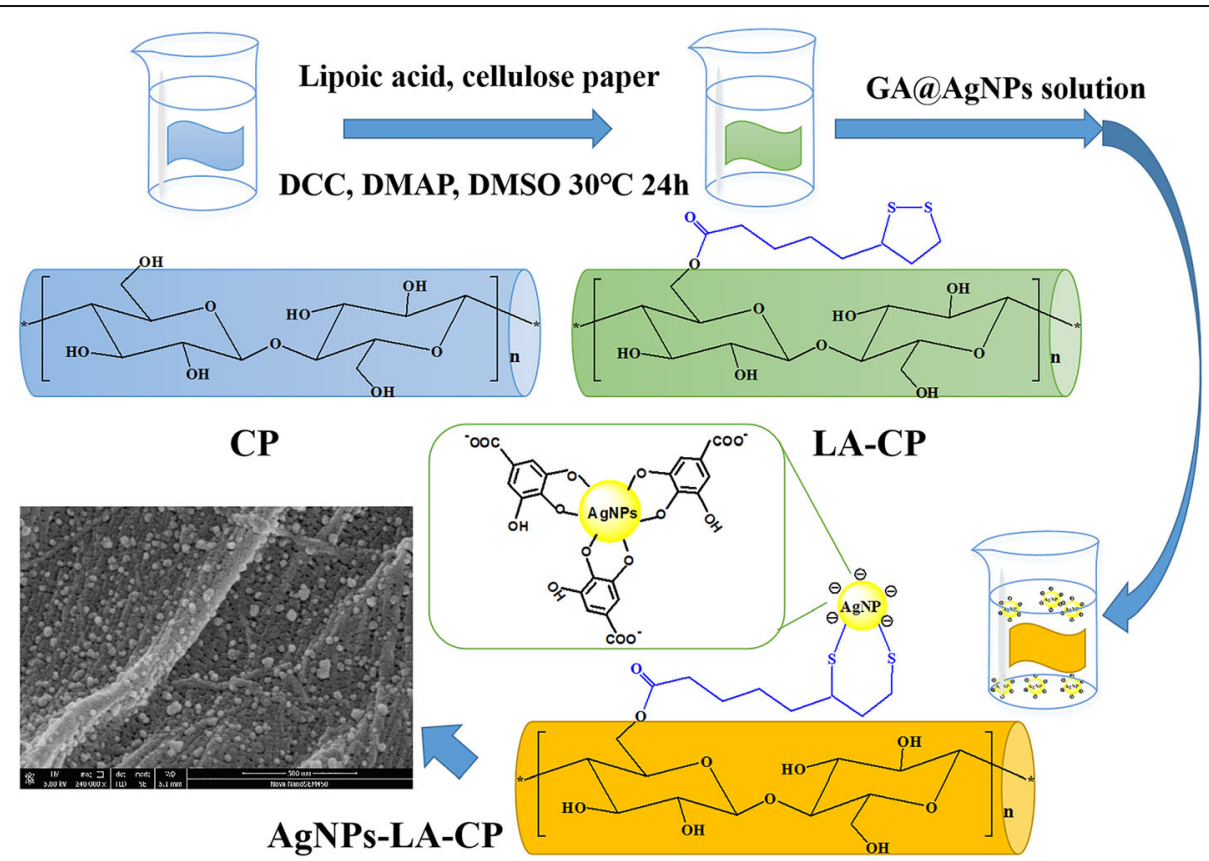

Fig. 1 Schematic illustration of the fabrication process of GA@AgNPs-LA-CP 

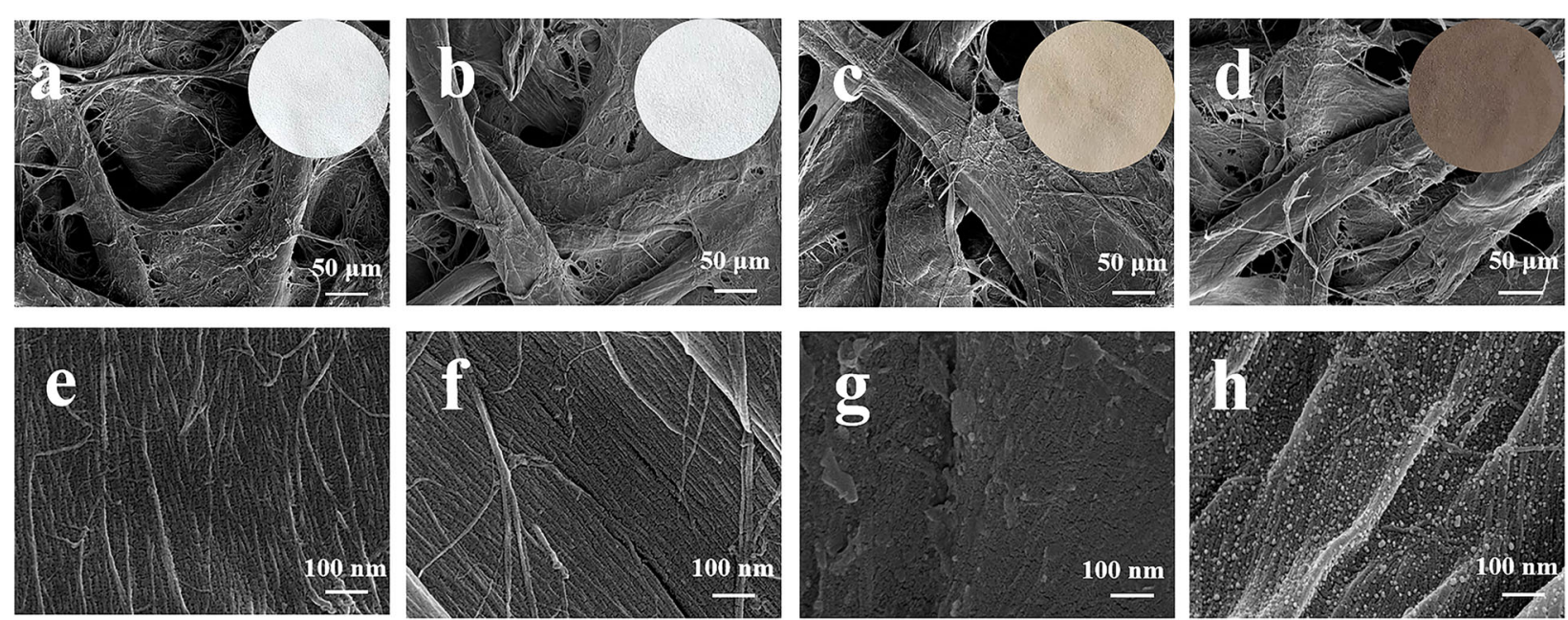

Fig. 2 SEM images of surface morphologies at different magnifications of $\mathbf{a}, \mathbf{e} C P, \mathbf{b}, \mathbf{f} L A-C P, \mathbf{c}, \mathbf{g}$ GA@AgNPs-CP, $\mathbf{d}, \mathbf{h}$ GA@AgNPs-LA-CP. The inset images in $\mathbf{a}, \mathbf{b}, \mathbf{c}$, and $\mathbf{d}$ are photographs of corresponding cellulose paper samples

paper was reacted with $5 \mathrm{~mL}$ of $70 \%$ nitric acid in $5 \mathrm{~mL}$ of water and then boiled until the paper disintegrated. After a few minutes of cooling, $5 \mathrm{~mL}$ of hydrogen peroxide (30\%) was added and the suspension was reboiled. After cooling, the suspension was diluted to $100 \mathrm{~mL}$ with water. Lastly, the $\mathrm{Ag}$ content in suspensions were tested by ICP-AES and the Ag content on paper was calculated.

\subsection{Catalytic reduction of 4-NP, MB and RhB}

To investigate the catalytic efficiency and reusability of the GA@AgNPs-LA-CP, the reduction of 4-NP was performed according to the previously reported procedure. In a typical experiment, $500 \mathrm{mg}$ GA@AgNPs-LA-CP was added into a mixed solution containing $35 \mathrm{~mL}$ deionized water and $7.5 \mathrm{~mL} 4-\mathrm{NP}$ aqueous solution $(3 \mathrm{mM})$. In experiment process, 500 mg GA@AgNPs-LA-CP was added into $20 \mathrm{~mL}$ each dye solution (MB, $10 \mathrm{mg} / \mathrm{L} ; \mathrm{RhB}, 10 \mathrm{mg} /$ L). $\mathrm{N}_{2}$ gas was then purged through the solution for 20 min to remove the dissolved $\mathrm{O}_{2}$. Subsequently, $5 \mathrm{ml}$ of freshly prepared $\mathrm{NaBH}_{4}$ solution $(0.3 \mathrm{M})$ was injected under continuous stirring to initiate the catalytic reduction reaction at $\mathrm{pH} 10$. At each time interval, part of the mixture was transferred into a quartz cuvette, and UV-vis absorption spectra were recorded in the range 250-550 $\mathrm{nm}(500-750 \mathrm{~nm} ; 450-650 \mathrm{~nm})$ to monitor the catalytic reaction of 4-NP (MB; RhB). The reaction was conducted at $295 \mathrm{~K}$, using a temperature-controlled water bath to keep the temperature at the $295 \mathrm{~K}$. Then, kinetic data was fitted with a pseudo-first-order model:

$$
\ln \left(C_{\mathrm{t}} / C_{0}\right)=\ln \left(A_{\mathrm{t}} / A_{0}\right)=-k_{\text {app }} t
$$

where $C_{0}$ and $C_{\mathrm{t}}$ represent the actual concentration of 4-
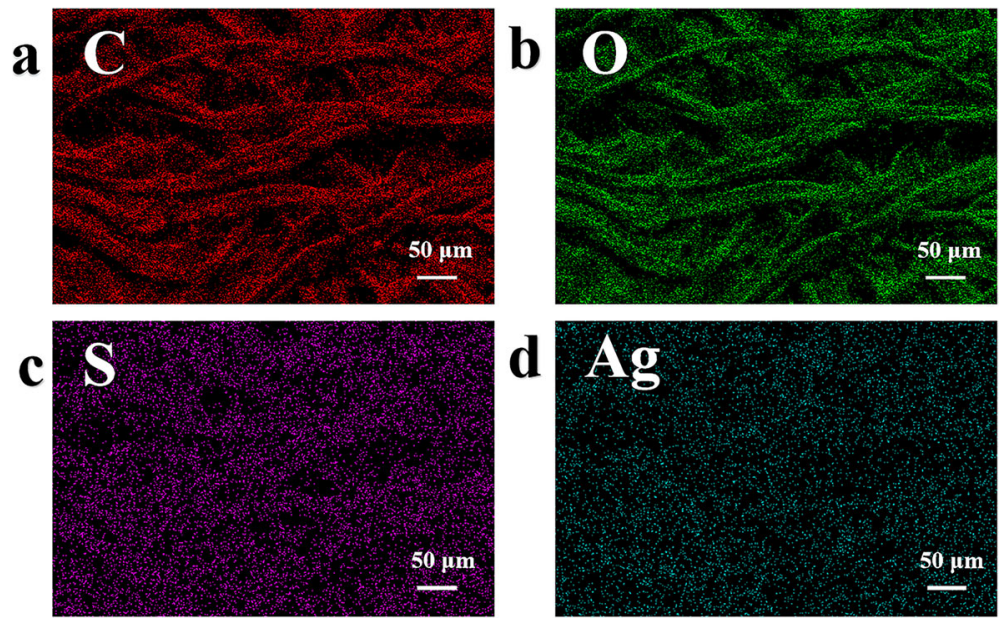

Fig. 3 EDS-mapping images a C, b O, c S, and $\mathbf{d}$ Ag of GA@AgNPs-LA-CP 

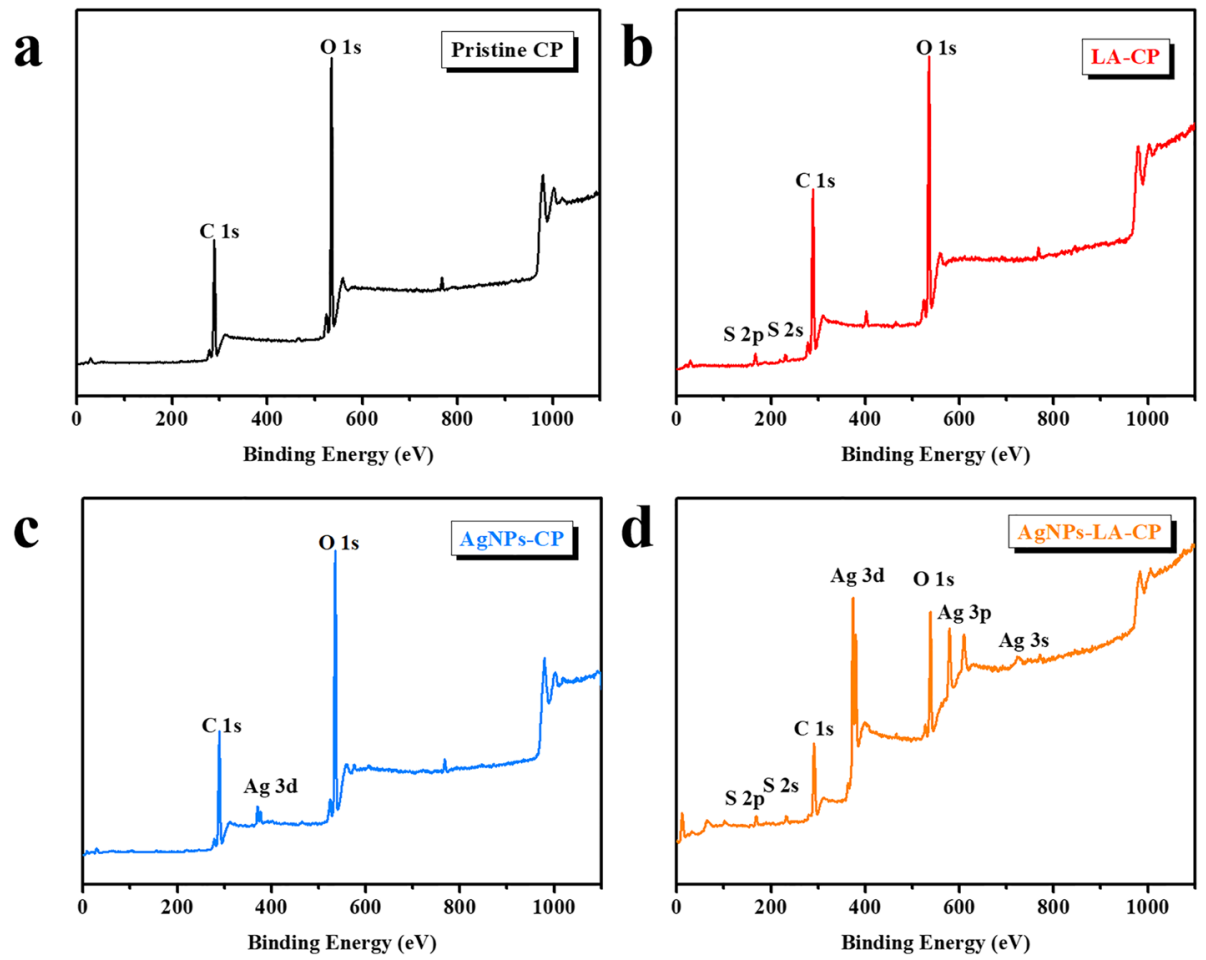

Fig. 4 XPS fully scanned spectra of a CP, b LA-CP, c GA@AgNPs-CP, and d GA@AgNPs-LA-CP

$\mathrm{NP}, \mathrm{MB}$, and $\mathrm{RhB}$, respectively, at the first time and at time $t$. In this experiment, the ratio of $C_{\mathrm{t}}$ to $C_{0}$ could be directly given by the ratio of the respective absorbances, $A_{\mathrm{t}} / A_{0}$. ( $A$ is the absorbance at 400,660 , and $555 \mathrm{~nm}$, respectively.) $k_{\text {app }}$ is the apparent first-order rate constant $\left(\mathrm{s}^{-1}\right)$. According to the apparent first-order rate equation (eq. 1$)$, when $\ln \left(A_{\mathrm{t}} / A_{0}\right)$ is proportional to time $t$, the reaction rate corresponds to the slope of the curve, $k_{\text {app }} . A$ linear relationship between $\ln \left(A_{\mathrm{t}} / A_{0}\right)$ and reaction time $t$ was observed, which matches first-order reaction kinetics.

\section{Results and discussion}

3.1 Preparation and characterization of GA@AgNPs-LA-CP Silver nanoparticles with an average diameter of $8 \mathrm{~nm}$ and several carboxyl groups on the surface were synthesized using gallic acid as the stabilizer, according to our previous work, which are defined as GA@AgNPs. In this

Table 1 Percentages of aliphatic $\mathrm{C}-\mathrm{C}, \mathrm{C}-\mathrm{O}, \mathrm{C}=\mathrm{O}$, and $\mathrm{O}-\mathrm{C}=\mathrm{O}$ bonds from XPS C1s peak fitting for pristine CP, LA-CP, GA@AgNPs-CP, and GA@AgNPs-LA-CP

\begin{tabular}{lllll}
\hline Simple & \multicolumn{5}{l}{ bond percentage (\%) } \\
\cline { 2 - 5 } & $\mathrm{C}-\mathrm{C}$ & $\mathrm{C}-\mathrm{O}$ & $\mathrm{C}=\mathrm{O}$ & $\mathrm{O}-\mathrm{C}=\mathrm{O}$ \\
\hline pristine CP & 51.5 & 48.5 & - & - \\
LA-CP & 28.6 & 43.8 & 20.9 & 6.7 \\
GA@AgNPs-CP & 49.4 & 50.6 & - & - \\
GA@AgNPs-LA-CP & 25.3 & 44.2 & 20.3 & 10.2 \\
\hline
\end{tabular}

work, as shown in Fig. 1, lipoic acid was selected to introduce the modification of cellulose fibers, with simultaneous incorporation of GA@AgNPs to form a GA@AgNPs-LA-CP. After washing, the resultant GA@AgNPs-LA-CP was brown, as shown in Fig. 2d. There were significant differences in the appearance of the paper before and after the deposition of GA@AgNPs. After being treated with GA@AgNPs solution for 30 min, the LA-CP color changed from white to brown, indicating the fixed state of GA@AgNPs on paper (the photographic images of LA-CP and GA@AgNPs-LA-CP are shown in Fig. 2b and d). Compared with the lightyellow color of GA@AgNPs-CP in Fig. 2c that formed only by the physical deposition of GA@AgNPs on the surface of cellulose papers, the brown color of GA@AgNPsLA-CP is considered to be inherited from the immobilization of GA@AgNPs due to the interaction between thiol and GA@AgNPs [46]. As illustrated in Fig. 1, the immobilization between thiol and GA@AgNPs on the surface of the cellulose fibers contributed to the lipoic acid modification (Fig. 2d), which may be stronger than the single physical deposition on cellulose fibers (Fig. 2c). Therefore, the GA@AgNPs on the surface of the cellulose fibers was investigated by SEM, using pristine cellulose papers (CP) and lipoic acid-modified cellulose paper (LA$\mathrm{CP}$ ) as the blank control (shown in Fig. 2a and b). In Fig. 2h, the quantity of GA@AgNPs on the surface of GA@AgNPs-LA-CP is significantly more than GA@AgNPs- 

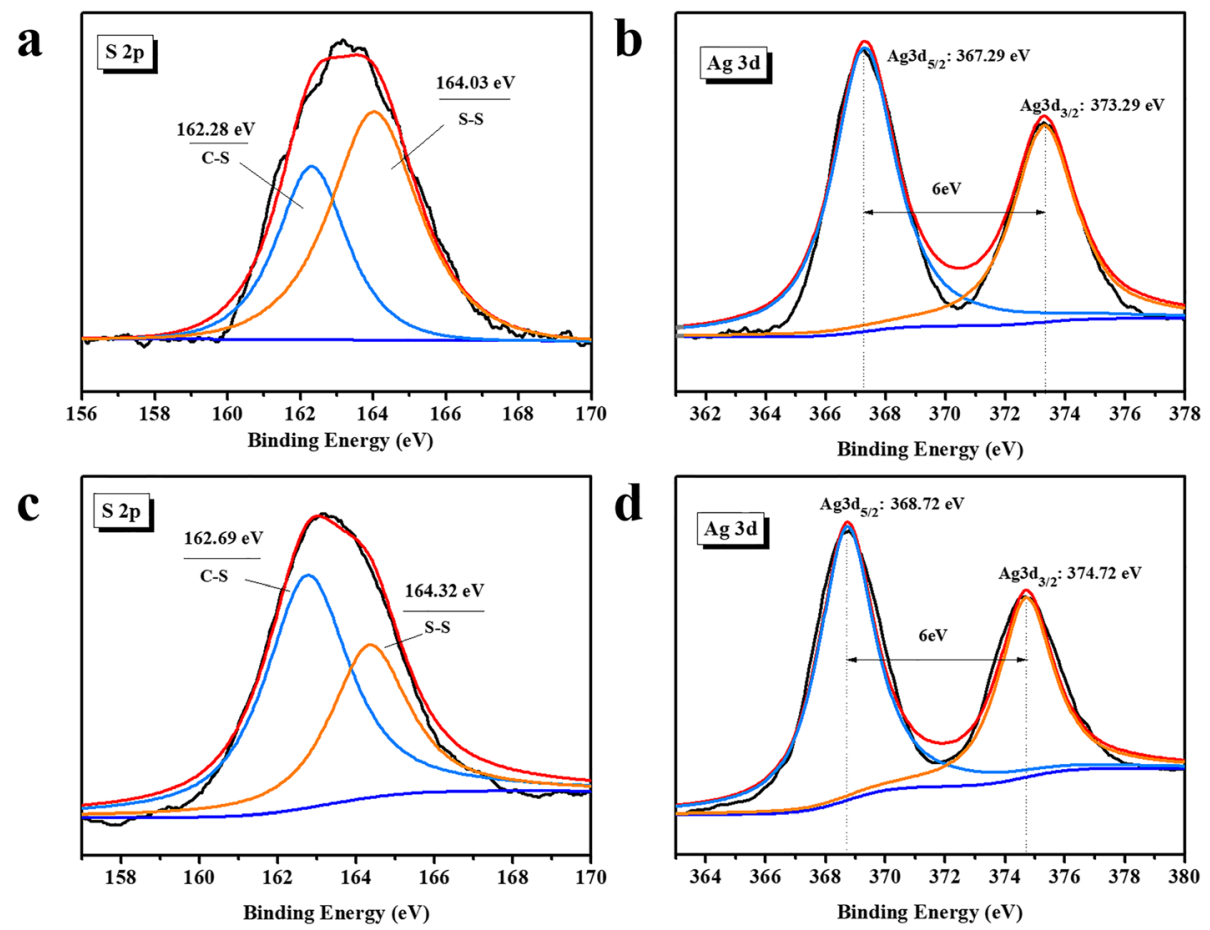

Fig. 5 XPS spectra of the samples in the S 2p region a CP, c LA-CP and in the Ag 3d region b GA@AgNPs-CP, d GA@AgNPs-LA-CP

a

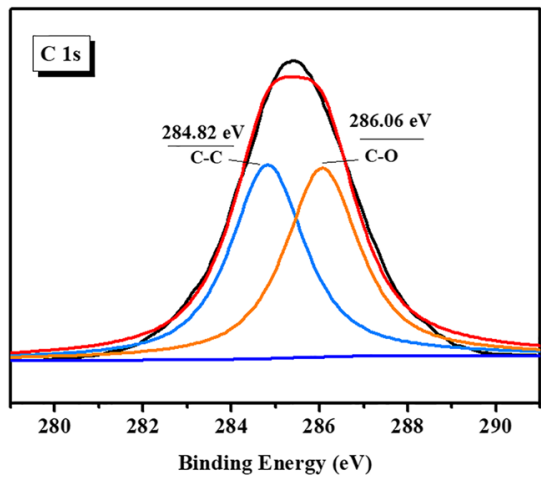

c

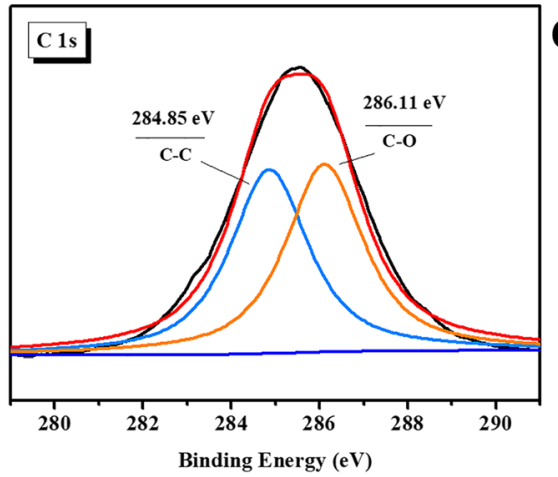

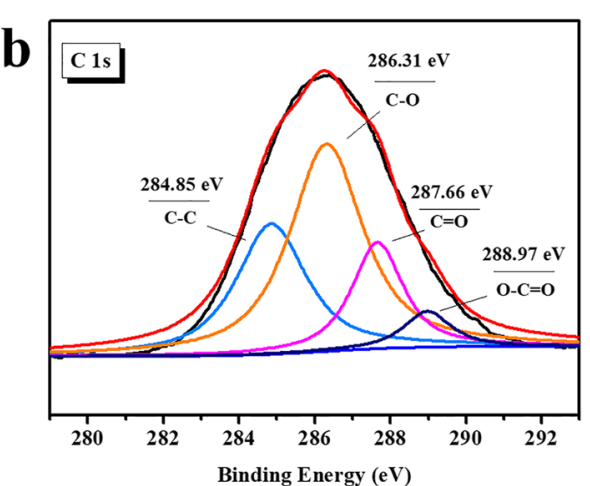

d

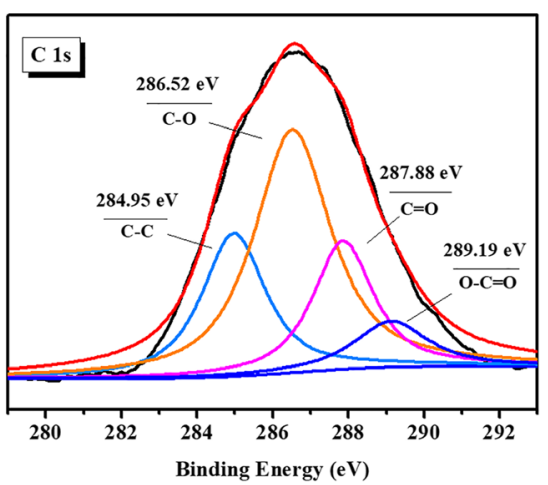

Fig. 6 XPS spectra of the samples in the C 1 s region a CP, b LA-CP, c GA@AgNPs-CP, and $\mathbf{d}$ GA@AgNPs-LA-CP 

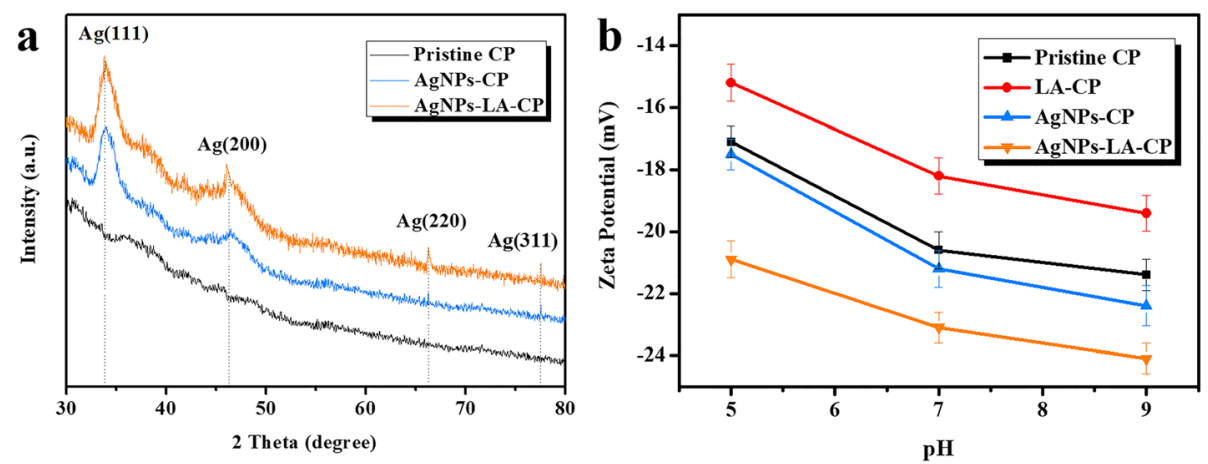

Fig. 7 a XRD patterns and $\mathbf{b}$ Surface $\zeta$-potential of different samples

LA-CP primarily immobilized by physical deposition. The results reveal several GA@AgNPs with multiple carboxyl groups immobilized on the surface of GA@AgNPs-LA-CP. The morphology of the GA@AgNPs-LA-CP and the distribution of GA@AgNPs on paper was directly observed with FESEM. The natural cellulosic structure and fibers on the surface of the paper are shown in the low-magnification SEM images (Fig. 2a-d). At the same time, in Fig. 2e-h, the complete network structure on the cellulose fiber surface can be observed through high magnification. These results showed that LA or GA@AgNPs had no damage or effect on the natural structure of the paper and could maintain the original properties of the cellulose paper. However, compared with the magnified image of cellulose fibers on the original cellulose paper (Fig. 2e), many evenly distributed GA@AgNPs can be observed on the cellulose fibers (Fig. 2h). To obtain better insight on the elemental distribution in different papers, EDS was performed on the above samples. As shown in Figure S1, EDS results indicate that $S$ and $\mathrm{Ag}$ atoms were successfully immobilized onto GA@AgNPs-LA-CP, and it is also confirmed that lipoic acid plays an important role in the uniform distribution of
GA@AgNPs, which is consistent with SEM results. Moreover, the $\mathrm{S}$ signal of the LA-CP samples was detected by EDS spectroscopy (Figure S1), proving the successful lipoic acid modification on cellulose paper surface. Furthermore, the EDS mapping of $\mathrm{C}, \mathrm{O}, \mathrm{S}$, and Ag elements in Fig. 3a-d also confirms the uniform distribution of GA@AgNPs on the surface of GA@AgNPs-LA-CP at low magnification. Since GA@AgNPs were immobilized onto cellulose fibers by lipoic acid modification in the GA@AgNPs-LA-CP, the silver loading content of the paper determined by ICP-AES was $6.7 \mathrm{mg}$ per gram of dry paper, which is higher than the value of $2.3 \mathrm{mg}$ per gram on GA@AgNPs-CP. These results verify that GA@AgNPs were immobilized onto cellulose fibers by thiol after the lipoic acid modification, leading to uniform distribution of GA@AgNPs on the interface of cellulose fibers. Meanwhile, signals measured by EDS mapping are beneficial to high loadings of GA@AgNPs.

XPS full spectra of pristine cellulose paper (CP) only showed peaks for $\mathrm{C} 1 \mathrm{~s}(286 \mathrm{eV})$ and $\mathrm{O} 1 \mathrm{~s}(531 \mathrm{eV})$ (Fig. 4a). Lipoic acid modification of the cellulose paper (LA-CP) surface was detected by the appearance of the $\mathrm{S}$

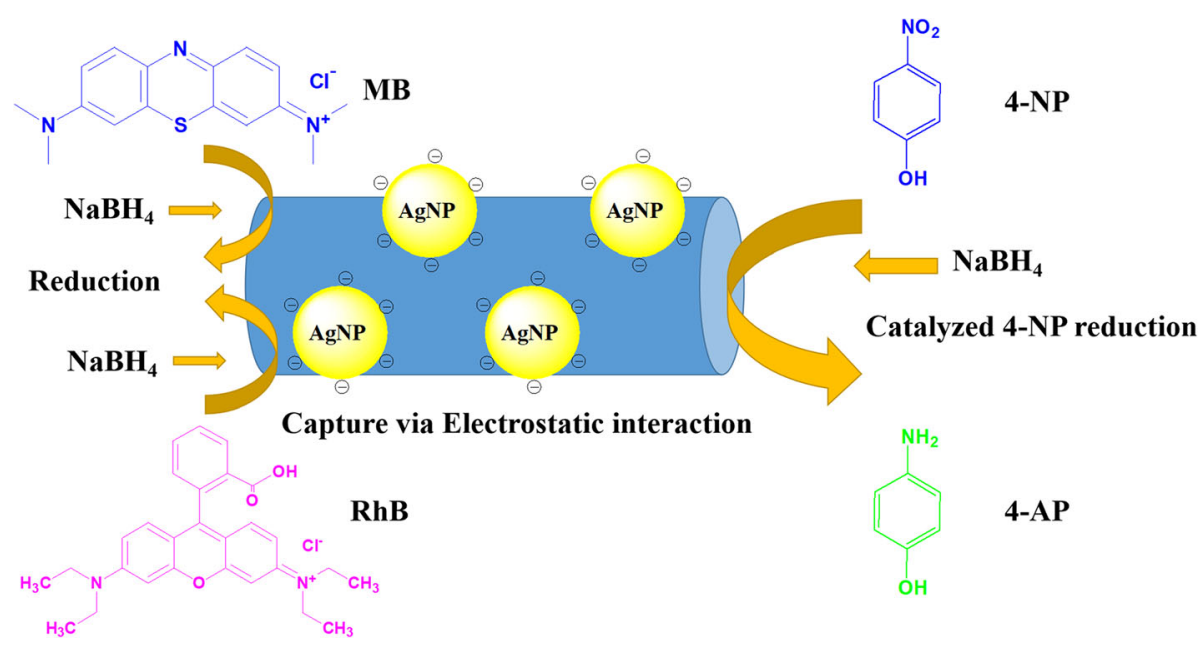

Fig. 8 Schematic illustration of the catalytic process via application of GA@AgNPs-LA-CP 

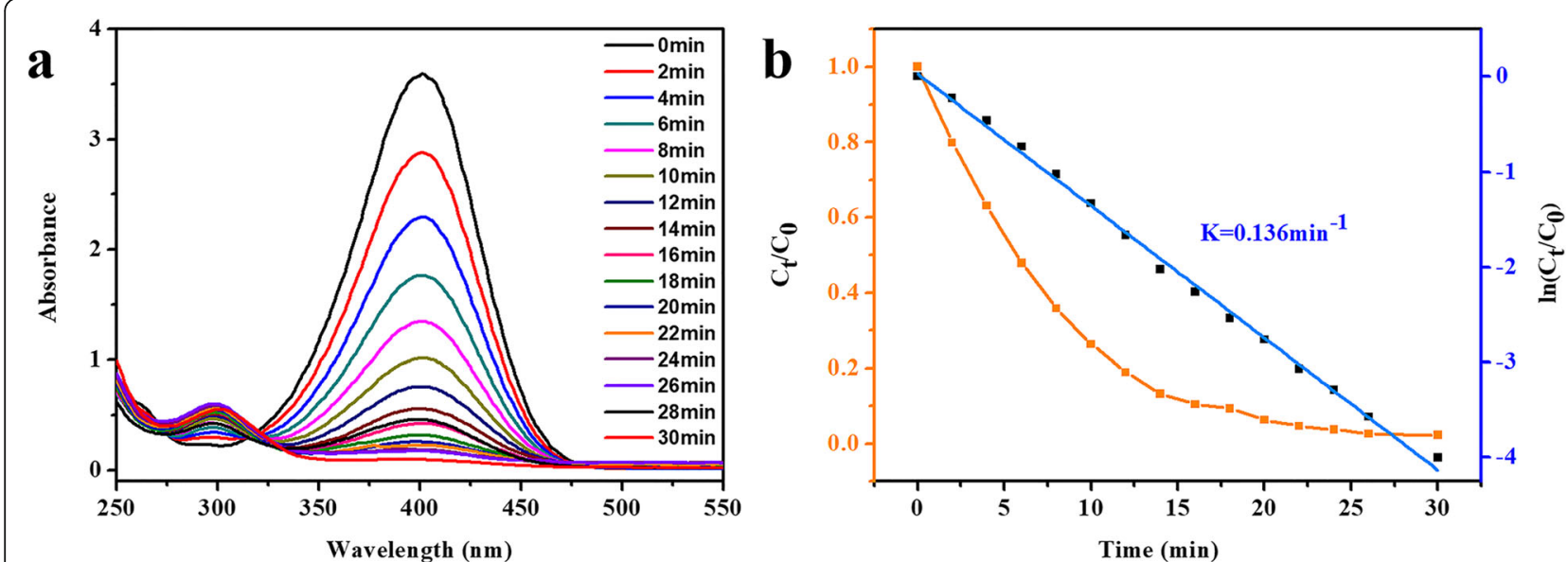

Fig. 9 Catalytic performance of GA@AgNPs-LA-CP for the catalysis of 4-NP to 4-AP. Time-dependent UV-vis spectral changes a and the reaction kinetics curve b

2p peak (163 eV) (Fig. 4b), and then the immobilization of GA@AgNPs on the LA-CP was confirmed by the appearance of the divisive doublet of $\mathrm{Ag} 3 \mathrm{~d}$ near $370 \mathrm{eV}$, $\mathrm{Ag} 3 \mathrm{p}$ at 571.8 and $602.6 \mathrm{eV}$, and $\mathrm{Ag} 3 \mathrm{~s}$ at $717.2 \mathrm{eV}$ (Fig. 4d). The Ag 3d spectrum shown in Fig. 5d consist of two distinct peak components with binding energies of $\mathrm{Ag}$ $3 \mathrm{~d}_{3 / 2}$ and $\mathrm{Ag} 3 \mathrm{~d}_{5 / 2}$ at 368.72 and $374.72 \mathrm{eV}$, respectively, both ascribed to the Ag0 species, which indicated the immobilization of GA@AgNPs. Moreover, the $6 \mathrm{eV}$ splitting of the $3 \mathrm{~d}$ doublet due to the spin-orbit coupling confirms the existence of the Ag0 state on the GA@AgNPs-LA-CP surface. Further characterization by XPS was performed to probe the surface functionalization of the cellulose paper, as illustrated in Fig. 1. The high-resolution C $1 \mathrm{~s}$ spectra of CP, LA-CP, GA@AgNPs-CP, and GA@AgNPs-LA-CP are shown in Fig. 6, panels a, b, c, and d, respectively. The peak of C $1 \mathrm{~s}$ XPS spectra of GA@AgNPs-LA-CP exhibited four peaks at $284.95 \mathrm{eV}$, $286.52 \mathrm{eV}, 287.88 \mathrm{eV}$, and $289.19 \mathrm{eV}$, which can be assigned to the saturated $\mathrm{C}-\mathrm{C}$ bonding of cellulose, the $\mathrm{C}-\mathrm{O}$ groups, carbonyl groups $(\mathrm{C}=\mathrm{O})$, and ester groups $(\mathrm{O}-\mathrm{C}=\mathrm{O})$, respectively. As cellulose is a natural biomass material [47], it shows the peaks for C-C $(284.82 \mathrm{eV})$ and $\mathrm{C}-\mathrm{O}(286.06 \mathrm{eV})$ for pristine $\mathrm{CP}$. The lipoic acid-modified cellulose paper (LA-CP) showed a significant changes at the peak position, and the peak of the high-resolution $\mathrm{C} 1 \mathrm{~s}$ peak showed four components which were assigned to $\mathrm{C}-\mathrm{C}(284.85 \mathrm{eV}), \mathrm{C}-\mathrm{O}(286.31 \mathrm{eV}), \mathrm{C}=\mathrm{O}$ $(287.66 \mathrm{eV})$, and $\mathrm{O}-\mathrm{C}=\mathrm{O}(288.97 \mathrm{eV})$. The new peak at 288.97 $\mathrm{eV}$ indicated the formation of $\mathrm{O}-\mathrm{C}=\mathrm{O}$ bonds because of the coupling reaction between the hydroxyl group of cellulose and the carboxyl group of lipoic acid. It was observed that the ratio of the chemical bonds $\mathrm{C}-\mathrm{O}$ originating from the cellulose structure, to $\mathrm{O}-\mathrm{C}=\mathrm{O}$, which was ascribed to lipoic acid modification, increased in the C $1 \mathrm{~s}$ XPS spectra of LA-CP (Fig. 4b). This results confirmed that lipoic acid modification was successfully accomplished. Next, the C 1s XPS spectra of the GA@AgNPs-LA-CP exhibited no major changes in the peak positions (Fig. 4d). The bond percentages of different bonds based on the $\mathrm{C} 1 \mathrm{~s}$ spectra for pristine CP, LACP, GA@AgNPs-CP, and GA@AgNPs-LA-CP are calculated in Table 1.

The X-ray diffraction patterns confirm the immobilization of GA@AgNPs on the surface of cellulose paper by the lipoic acid modification method (Fig. 7a). The largest peaks were observed from GA@AgNPs-LA-CP. Furthermore, compared to the XRD curves of CP samples without other crystal diffraction peaks, for both GA@AgNPs-CP and GA@AgNPsLA-CP, The Bragg reflection peaks of $2 \theta$ arise at $33.5^{\circ}$, $46.4^{\circ}, 66.8^{\circ}$, and $77.5^{\circ}$, which are coincident with the $(11$

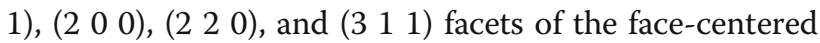
cubic structure of crystalline $\mathrm{Ag}$ and indicate the immobilization of GA@AgNPs on cellulose fibers, respectively $[41,48]$. Lower peak intensities were observed in the GA@AgNPs-CP samples and most peaks were in similar locations. No peaks were observed for the pristine cellulose paper. To further understand the surface charge of GA@AgNPs-LA-CP, the surface $\zeta$-potential of four cellulose papers were analyzed under water environments with three different $\mathrm{pH}$ values. In Fig. $7 \mathrm{~b}$, pristine cellulose paper exhibits a negative surface with a zeta-potential of $-20.6 \pm 0.46 \mathrm{mV}$ at $\mathrm{pH} 7$ due to a large number of $-\mathrm{OH}$ groups on the cellulose fibers $[49,50]$. By contrast, the GA@AgNPs-LA-CP shows a more negative charge $(-23.5 \pm 0.51 \mathrm{mV})$, which is ascribed to the immobilization of several GA@AgNPs with negatively charged anionic carboxyl groups. As illustrated in Fig. 8, the resultant negatively charged surface can adsorb more cationic dyes through electrostatic attractive forces.

\section{Catalytic properties of GA@AgNPs-LA-CP}

The catalytic activities of the GA@AgNPs-LA-CP was tested via the catalysis of 4-NP. The catalysis from 4-NP 

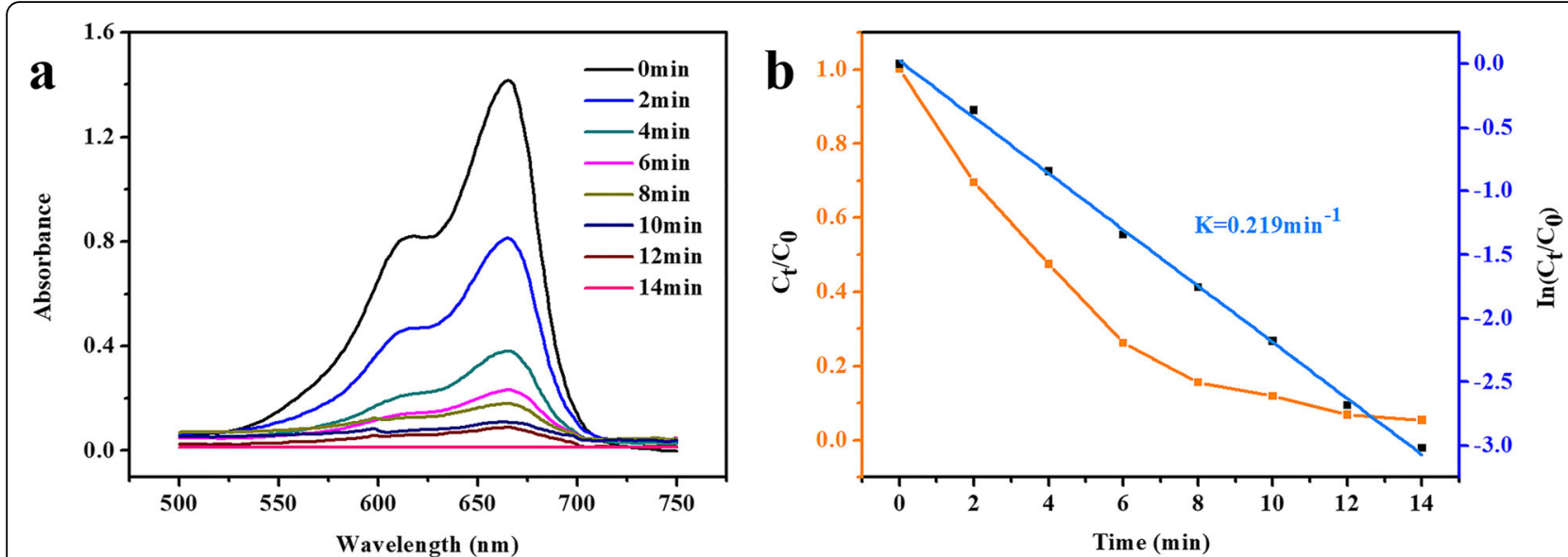

Fig. 10 Catalytic performance of GA@AgNPs-LA-CP for the catalysis of MB. Time-dependent UV-vis spectral changes a and the reaction kinetics curve $\mathbf{b}$

to 4-AP was difficult to proceed without the catalyst, which is ascribe to the absence of electronic relay stations between the reactants and products of catalysis $[41,51]$. Whereas, as shown in Fig. 8, AgNPs could enhance the catalysis as an electronic relay station to overcome the electronic obstacles in the catalytic process, which can transfer the electron from AgNPs to 4-NP. As mentioned in many previous papers [13], the absorption peak of 4-NP in $\mathrm{NaBH}_{4}$ aqueous solution is $400 \mathrm{~nm}$. Many studies $[15,45]$ have reported that during the catalytic process, the absorption peak strength at $400 \mathrm{~nm}$ gradually decreased and the absorption peak strength at $300 \mathrm{~nm}$ increased, which corresponds to the yellowgreen fading of 4-nitrophenol and the formation of 4AP. The catalysis process were recorded by UV-vis-NIR spectrophotometer with the absorption bands of 250$550 \mathrm{~nm}$. At the same time, 4-AP, which is the product of the catalysis, is a useful industrial chemicals for dye production and biomedical applications. The catalysis reaction was rapid and the solution became colorless within 30 min after the GA@AgNPs-LA-CP were added to the 4NP solution, which is consistent with the results of UV-vis spectra in Fig. 9. The catalytic reaction is quantitatively verified by the changes in the absorbance of UV-vis spectra.

The catalysis performance for cation dyes of the GA@AgNPs-LA-CP was tested and measured by adding the GA@AgNPs-LA-CP paper pieces in methylene blue (MB) and Rhodamine B (RhB) solutions, respectively. In experiment, the two cation dyes were tested, which may testify the practical catalytic applications for tannery wastewater. Figures 10 and 11 demonstrate the dye removal ratio and removal rate with time for two cation dyes under the catalysis of GA@AgNPs-LA-CP. The dye removal ratio of $\mathrm{MB}$ is almost $100 \%$ and fades colorless in $14 \mathrm{~min}$, which could prove high efficiency of GA@AgNPs-LA-CP as a catalyst. In addition, the
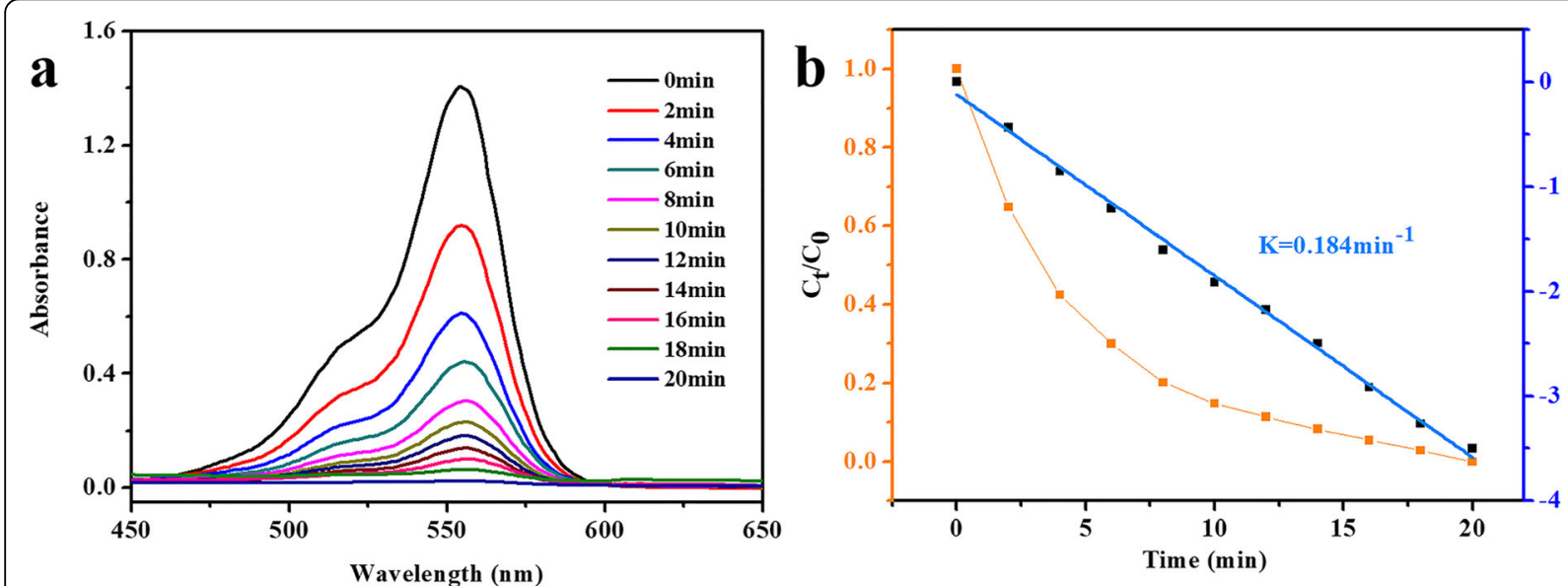

Fig. 11 Catalytic performance of GA@AgNPs-LA-CP for the catalysis of RhB. Time-dependent UV-vis spectral changes a and the reaction kinetics curve $\mathbf{b}$ 
catalytic kinetic experiments of the GA@AgNPs-LA-CP were tested with the two cation dyes, as is shown in Fig. 10. The GA@AgNPs-LA-CP indicated a high efficiency catalytic applications for the two cation dyes of tannery wastewater approximately. The high efficiency of GA@AgNPs-LA-CP as a catalyst benefit from cellulose fiber network structure and the uniform immobilization of GA@AgNPs on the surface of cellulose paper, which is especially on account of the electrostatic forces and hydrogen bonding between cation dyes and GA@AgNPs-LA-CP. Furthermore, the data calculation model is listed to facilitate the analysis of its catalytic performance. Furthermore, $k_{\text {app }}$ values for the reactions with 4-NP, MB, and $\mathrm{RhB}$ were calculated to be $0.136,0.219$, and $0.184 \mathrm{~min}^{-1}$. The $k_{\text {nor }}$ values of $4-\mathrm{NP}$, $\mathrm{MB}$, and $\mathrm{RhB}$, which is on behalf of $k_{\mathrm{app}}$ values after the normalization by the amount of GA@AgNPs-LA-CP were $0.272,0.438$, and $0.368 \mathrm{~min}^{-1} \mathrm{~g}^{-1}$, respectively.

\section{Conclusion}

Facile, efficient, and robust immobilization of GA@AgNPs on cellulose paper is a useful method in materials chemistry and catalytic applications. In this work, a facile lipoic acid modification method for the immobilization of GA@AgNPs on cellulose paper was developed. GA@AgNPs were uniformly distributed on the surface of cellulose paper without aggregation. The facile fabrication of a catalytic substrate with a 3D porous cellulose fiber network has advantages of abundance and low cost for practical wastewater treatment applications. Moreover, the GA@AgNPs-LA-CP exhibited good catalytic activity for 4-NP, MB, and RhB. Given the interwoven cellulosic fiber structure, lipoic acid modification, and low cost of cellulose papers, immobilization of GA@AgNPs onto the surface of cellulose fibers shows great potential for tannery wastewater treatment applications. Using a simple, low cost, environmentally friendly composite catalyst is novel and provides new avenues for this technology.

\section{Supplementary information}

Supplementary information accompanies this paper at https://doi.org/10. 1186/s42825-020-00019-y.

Additional file 1: Figure S1. EDS spectra of a $C P, b L A-C P, c$ GA@AgNPs-CP, d GA@AgNPs-LA-CP. Figure S2. XPS spectra of the samples in the 01 s region a CP, b LA-CP, c GA@AgNPs-CP, d GA@AgNPsLA-CP.

\section{Abbreviations}

GA: Gallic acid; AgNPs: Silver nanoparticles; LA: Lipoic acid; CP: Cellulose papers; SEM: Scanning electron microscope

\section{Acknowledgements}

This work was supported by the support program of Chengdu Science and Technology Bureau (2019-GH02-00001-HZ), opening project of State Key Laboratory of Hydraulics and Mountain River Engineering, Sichuan University (SKHL1816) and opening project of Key Laboratory of Leather Chemistry and Engineering of Ministry of Education, Sichuan University (20826041C4159).

\section{Authors' contributions}

LG conceived and designed the study. YR and LT performed the experiments and analyzed the experiments data. YR, PH and JJ reviewed and edited the manuscript. All authors read and approved the manuscript.

\section{Availability of data and materials}

All data generated or analyzed during this study are included in this published article. The authors declare that the data in this article is reliable.

\section{Competing interests}

The authors declare that they have no competing interests.

\section{Author details}

${ }^{1}$ National Engineering Laboratory for Clean Technology of Leather Manufacture, College of Biomass Science and Engineering, Sichuan University, Chengdu 610065, China. ${ }^{2}$ The Key Laboratory of Leather Chemistry and Engineering of Ministry of Education, College of Biomass Science and Engineering, Sichuan University, Chengdu 610065, China. ${ }^{3}$ The State Key Laboratory of Hydraulic and Mountain River Engineering, Sichuan University, Chengdu 610065, China.

Received: 3 December 2019 Accepted: 4 March 2020 Published online: 20 March 2020

\section{References}

1. He C, Cheng J, Zhang X, Douthwaite M, Pattisson S, Hao Z. Recent advances in the catalytic oxidation of volatile organic compounds: a review based on pollutant sorts and sources. Chem Rev. 2019;119:4471-568.

2. Gawande MB, Goswami A, Asefa T, Guo H, Biradar AV, Peng DL, Zboril R, Varma RS. Core-shell nanoparticles: synthesis and applications in catalysis and electrocatalysis. Chem Soc Rev. 2015;44:7540-90.

3. Song $Y$, Jiang $H$, Wang $B$, Kong $Y$, Chen J. Silver-incorporated mussel-inspired polydopamine coatings on mesoporous silica as an efficient nanocatalyst and antimicrobial agent. ACS Appl Mater Interfaces. 2018;10:1792-801.

4. Ramalingam B, Khan MMR, Mondal B, Mandal AB, Das SK. Facile synthesis of silver nanoparticles decorated magnetic-chitosan microsphere for efficient removal of dyes and microbial contaminants. ACS Sustain Chem Eng. 2015;3:2291-302.

5. Kim JG, Cha MC, Lee J, Choi T, Chang JY. Preparation of a sulfurfunctionalized microporous polymer sponge and in situ growth of silver nanoparticles: a compressible monolithic catalyst. ACS Appl Mater Interfaces. 2017:9:38081-8.

6. Dolatkhah A, Jani P, Wilson LD. Redox-responsive polymer template as an advanced multifunctional catalyst support for silver nanoparticles. Langmuir. 2018;34:10560-8.

7. Das SK, Dickinson C, Lafir F, Broughamc DF, Marsili E. Synthesis, characterization and catalytic activity of gold nanoparticles biosynthesized with Rhizopus oryzaeprotein extract. Green Chem. 2012;14:1322.

8. Jiao T, Zhao H, Zhou J, Zhang Q, Luo X, Hu J, Peng Q, Yan X. Self-assembly reduced graphene oxide nanosheet hydrogel fabrication by anchorage of chitosan/silver and its potential efficient application toward dye degradation for wastewater treatments. ACS Sustain Chem Eng. 2015;3:3130-9.

9. Xie $Y$, Yan $B, X u H$, Chen J, Liu $Q$, Deng $Y$, Zeng $H$. Highly regenerable mussel-inspired $\mathrm{Fe}_{3} \mathrm{O}_{4} @$ polydopamine-Ag core-shell microspheres as catalyst and adsorbent for methylene blue removal. ACS Appl Mater Interfaces. 2014;6:8845-52.

10. Das SK, Parandhaman T, Pentela N, Islam AKMM, Mandal AB, Mukherjee M. Understanding the biosynthesis and catalytic activity of Pd, Pt, and $\mathrm{Ag}$ nanoparticles in hydrogenation and Suzuki coupling reactions at the Nanobio Interface. J Phys Chem C. 2014;118:24623-32.

11. Peng N, Hu D, Zeng J, Li Y, Liang L, Chang C. Superabsorbent cellulose-Clay nanocomposite hydrogels for highly efficient removal of dye in water. ACS Sustain Chem Eng. 2016;4:7217-24.

12. Parandhaman T, Pentela N, Ramalingam B, Samanta D, Das SK. Metal nanoparticle loaded magnetic-chitosan microsphere: water dispersible and 
easily separable hybrid metal Nano-biomaterial for catalytic applications. ACS Sustain Chem Eng. 2017:5:489-501.

13. Song J, Zhu Y, Zhang J, Yang J, Du Y, Zheng W, Wen C, Zhang Y, Zhang L. Encapsulation of AgNPs within zwitterionic hydrogels for highly efficient and antifouling catalysis in biological environments. Langmuir. 2019;35:1563-70.

14. Das SK, Khan MMR, Guha AK, Naskar N. Bio-inspired fabrication of silver nanoparticles on nanostructured silica: characterization and application as a highly efficient hydrogenation catalyst. Green Chem. 2013;15:2548.

15. Li H, Jiang D, Huang Z, He K, Zeng G, Chen A, Yuan L, Peng M, Huang T, Chen G. Preparation of silver-nanoparticle-loaded magnetic biochar/poly (dopamine) composite as catalyst for reduction of organic dyes. J Colloid Interface Sci. 2019;555:460-9.

16. Wang J, Liu W, Li H, Wang H, Wang Z, Zhou W, Liu H. Preparation of cellulose fiber- $\mathrm{TO}_{2}$ nanobelt-silver nanoparticle hierarchically structured hybrid paper and its photocatalytic and antibacterial properties. Chem Eng J. 2013;228:272-80.

17. Bootharaju MS, Pradeep T. Facile and rapid synthesis of a Dithiol-protected $\mathrm{Ag}_{7}$ quantum cluster for selective adsorption of cationic dyes. Langmuir. 2013;29:8125-32.

18. Liu G, Yu R, Lan T, Liu Z, Zhang P, Liang R. Gallic acid-functionalized graphene hydrogel as adsorbent for removal of chromium (III) and organic dye pollutants from tannery wastewater. RSC Adv. 2019;9:27060-8.

19. Liu C, Ye X, Wang X, Liao X, Huang X, Shi B. Collagen fiber membrane as an absorptive substrate to coat with carbon nanotubes-encapsulated metal nanoparticles for lightweight, wearable, and absorption-dominated shielding membrane. Ind Eng Chem Res. 2017;56:8553-62.

20. Li K, Yu R, Zhu R, Liang R, Liu G, Peng B. pH-sensitive and chromium-loaded mineralized nanoparticles as a tanning agent for cleaner leather production. ACS Sustain Chem Eng. 2019;7:8660-9.

21. Lee HJ, Cho W, Lim E, Oh M. One-pot synthesis of magnetic particleembedded porous carbon composites from metal-organic frameworks and their sorption properties. Chem Commun. 2014;50:5476-9.

22. Zhuang Z, Yang Q, Chen W. One-step papid and facile synthesis of subnanometer-sized $\mathrm{Pd}_{6}\left(\mathrm{C}_{12} \mathrm{H}_{25} \mathrm{~S}\right)_{11}$ clusters with ultra-high catalytic activity for 4-nitrophenol reduction. ACS Sustain Chem Eng. 2019;7:2916-23.

23. Miao L, Liu G, Wang J. Ag-nanoparticle-bearing poly (vinylidene fluoride) nanofiber mats as janus filters for catalysis and separation. ACS Appl Mater Interfaces. 2019;11:7397-404.

24. Yin S, Tian H, Ren Z, Wei X, Chao C, Pei J, Li X, Xu G, Shen G, Han G. Octahedral-shaped perovskite nanocrystals and their visible-light photocatalytic activity. Chem Commun. 2014;50:6027-30

25. Tzounis L, Doña M, Lopez-Romero JM, Fery A, Contreras-Caceres R. Temperature-controlled catalysis by core-shell-satellite AuAg@pNIPAM@Ag hybrid microgels: a highly efficient catalytic thermoresponsive nanoreactor. ACS Appl Mater Interfaces. 2019;11:29360-72.

26. Zhang $\mathrm{CL}$, Yu SH. Nanoparticles meet electrospinning: recent advances and future prospects. Chem Soc Rev. 2014:43:4423-48.

27. Huang T, Shao Y, Zhang Q, Deng Y, Liang Z, Guo F, Li P, Wang Y. Chitosancross-linked graphene oxide/carboxymethyl cellulose aerogel globules with high structure stability in liquid and extremely high adsorption ability. ACS Sustain Chem Eng. 2019;7:8775-88.

28. Liu K, Cheng P, Kong C, Guo Q, Lu Z, Liu Q, Li M, Wang WW, Wang Y, Zhong $W$, Sun $G$, Wang $D$. A readily accessible functional nanofibrous membrane for high-capacity immobilization of Ag nanoparticles and ultrafast catalysis application. Adv Mater Interfaces. 2019;6:1801617.

29. Chen M, Xiao C, Wang C, Liu H, Huang H, Yan D. Fabrication of tubular braid reinforced PMIA nanofiber membrane with mussel-inspired Ag nanoparticles and its superior performance for the reduction of 4nitrophenol. Nanoscale. 2018;10:19835-45.

30. Luo G, Jiang $X$, Li M, Shen $Q$, Zhang $L$, Yu H. Facile fabrication and enhanced photocatalytic performance of $\mathrm{Ag} / \mathrm{AgCl} / \mathrm{rGO}$ heterostructure photocatalyst. ACS Appl Mater Interfaces. 2013;5:2161-8.

31. Liu G, Han K, Zhou Y, Ye H, Zhang X, Hu J, Li X. Facile synthesis of highly dispersed Ag doped graphene oxide/Titanate nanotubes as a visible light photocatalytic membrane for water treatment. ACS Sustain Chem Eng. 2018;6:6256-63.

32. Vilardi G, Rodríguez-Rodríguez J, Ochando-Pulido JM, Verdone N, MartinezFerez A, Palma LD. Large laboratory-plant application for the treatment of a tannery wastewater by Fenton oxidation: Fe (II) and nZVI catalysts comparison and kinetic modelling. Process Saf Environ Prot. 2018;117:629-38.

33. Vilardi G, Ochando-Pulido JM, Stoller M, Verdone N, Palma LD. Fenton oxidation and chromium recovery from tannery wastewater by means of iron-based coated biomass as heterogeneous catalyst in fixed-bed columns. Chem Eng J. 2018:351:1-11.

34. Yang Z, Guo H, Yao Z, Mei Y, Tang CY. Hydrophilic silver nanoparticles induce selective nanochannels in thin film nanocomposite polyamide membranes. Environ Sci Technol. 2019;53:5301-8.

35. Horecha M, Kaul E, Horechyy A, Stamm M. Polymer microcapsules loaded with Ag nanocatalyst as active microreactors. J Mater Chem A. 2014;2:7431-8.

36. Vilardi G, Rodriguez-Rodriguez J, Ochando-Pulido JM, Palma LD, Verdone N. Fixed-bed reactor scale-up and modelling for $\mathrm{Cr}(\mathrm{VI})$ removal using nano ironbased coated biomass as packing material. Chem Eng J. 2019;361:990-8.

37. Huang PT, Chen YN, Chen KC, Wuan SH, Liu CP. Confinement of silver nanoparticles in polystyrenes through molecular entanglements and their application for catalytic reduction of 4-nitrophenol. J Mater Chem A. 2019;7:20919-25.

38. Mu B, Wang $Q$, Wang A. Preparation of magnetic attapulgite nanocomposite for the adsorption of $\mathrm{Ag}^{+}$and application for catalytic reduction of 4-nitrophenol. J Mater Chem A. 2013;1:7083-90.

39. Xiang J, Ma L, Su H, Xiong J, Li K, Xia Q, Liu G. Layer-by-layer assembly of antibacterial composite coating for leather with cross-link enhanced durability against laundry and abrasion. Appl Surf Sci. 2018;458:978-87.

40. Liu G, Li K, Luo Q, Wang H, Zhang Z. PEGylated chitosan protected silver nanoparticles as water-borne coating for leather with antibacterial property. J Colloid Interface Sci. 2017:490:642-51.

41. Lan T, An R, Liu Z, Li K, Xiang J, Liu G. Facile fabrication of a biomass-based film with interwoven fibrous network structure as heterogeneous catalysis platform. J Colloid Interface Sci. 2018;532:331-42.

42. Xia Q, Yang L, Hu K, Li K, Xiang J, Liu G, Wang Y. Chromium cross-linking based immobilization of silver nanoparticle coating on leather surface with broad-spectrum antimicrobial activity and durability. ACS Appl Mater Interfaces. 2019;11:2352-63.

43. Ye X, Wang Y, Ke L, Cui Y, Luo W, Wang X, Huang X, Shi B. Competitive adsorption for simultaneous removal of emulsified water and surfactants from mixed surfactant-stabilized emulsions with high flux. J Mater Chem A. 2018;6:14058-64.

44. Tarannum N, Divya, Gautam YK. Facile green synthesis and applications of silver nanoparticles: a state-of-the-art review. RSC Adv. 2019;9:34926-48.

45. Liang M, Su R, Huang R, Qi W, Yu Y, Wang L, He Z. Facile in situ synthesis of silver nanoparticles on procyanidin-grafted eggshell membrane and their catalytic properties. ACS Appl Mater Interfaces. 2014;6:4638-49.

46. Vieillard J, Bouazizi N, Morshed MN, Clamens T, Desriac F, Bargougui R, Thebault P, Lesouhaitier O, Derf FL, Azzouz A. CuO nanosheets modified with amine and thiol grafting for high catalytic and antibacterial activities. Ind Eng Chem Res. 2019;58:10179-89.

47. Liu G, Xiang J, Xia Q, Li K, Lan T, Yu L. Superhydrophobic cotton gauze with durably antibacterial activity as skin wound dressing. Cellulose. 2019;26:1383-97.

48. Gao WY, Hao YC, Su X, Chen LW, Bu TA, Zhang N, Yu ZL, Zhu Z, Yin AX. Morphology-dependent electrocatalytic nitrogen reduction on Ag triangular nanoplates. Chem Commun. 2019;55:10705-8.

49. Adhikari L, Larm NE, Wagle DV, Atwood JL, Baker GA. Facile, one-pot, in aqua synthesis of catalytically competent gold nanoparticles using pyrogallol[4] arene as the sole reagent. Chem Commun. 2019;55:6261-4.

50. Islam MS, Akter N, Rahman MM, Shi C, Islam MT, Zeng H, Azam MS. Musselinspired immobilization of silver nanoparticles toward antimicrobial cellulose paper. ACS Sustain Chem Eng. 2018;6:9178-88.

51. Larm NE, Madugula D, Lee MW, Baker GA. Polyhedral borane-capped coinage metal nanoparticles as high-performing catalysts for 4-nitrophenol reduction. Chem Commun. 2019;55:7990-3.

\section{Publisher's Note}

Springer Nature remains neutral with regard to jurisdictional claims in published maps and institutional affiliations. 